\title{
An Improved Method for the Numerical Solution of the Suspension Bridge Deflection Equations*
}

\author{
By R. W. Dickey
}

1. Introduction. The purpose of this paper is to indicate an improved method for obtaining numerical solutions of the suspension bridge deflection equations.

For simplicity consider a suspension bridge consisting of a single span of length $l$. The vertical deflection of the roadway $w(x)$ satisfies the ordinary differential equation (cf. [1, pp. 75ff]):

$$
\frac{d^{2}}{d x^{2}} E I \frac{d^{2} w}{d x^{2}}-(H+h) \frac{d^{2} w}{d x^{2}}=P(x)-\frac{h}{H} Q(x)
$$

with boundary conditions

$$
w(0)=w(l)=0
$$

and

$$
w^{\prime \prime}(0)=w^{\prime \prime}(l)=0
$$

(the prime indicates differentiation with respect to $x$ ). $P(x)$ and $Q(x)$ (positive downward) are the live load per unit length and the dead load of the roadway, cable, and hangers per unit length. $E$ and $I$ (assumed constants) are the modulus of elasticity and moment of inertia of the roadway and $H$ (constant) is the horizontal dead-load tension in the cable. The term $h$ (constant), the induced live-load tension, depends on the vertical deflection $w(x)$ as follows (cf. [1]):

$$
h=\frac{E_{c} A_{c}}{H l_{c}} \int_{0}^{l} Q(x) w(x) d x
$$

where $E_{\mathrm{c}}$ is the modulus of elasticity of the cable, $A_{c}$ is the cross-sectional area of the cable, and $l_{c}$ is the length of the cable. Thus the Eq. (1.1) is nonlinear in $w$.

Heller, Isaacson, and Stoker [2] treated the Eqs. (1.1) and (1.3) with boundary conditions (1.2) by solving a sequence of linear problems. Thus (1.1) was first solved with $h=0$, and the solution of (1.1) was used in conjunction with (1.3) to calculate a new value of $h$. This new value of $h$ was placed in Eq. (1.1) and (1.1) was resolved for $w$. This process was then repeated. Although this iteration scheme converged, it converged to a solution with $H+h<0$, i.e. the cable was in compression. $\dagger$ The existence of these physically unrealistic 'compressive' solutions

Received June 2, 1967.

* This paper represents results obtained at the Courant Institute of Mathematical Sciences, New York University, under the sponsorship of the U. S. Army Research Office-Durham, Contract DA-31-124-ARO-D-360.

$\dagger$ In [2] it was shown that in the case of constant live and dead loads there were infinitely many 'compressive' solutions, but only one solution with $H+h>0$, i.e. corresponding to a tension in the cable. 
forced the authors of [2] to introduce an extrapolation factor-depending in a complicated way on the loads and physical characteristics of the suspension bridgeso that at each step the cable was in tension. In this way a solution was obtained with $H+h>0$.

In Section 2 of this paper the difficulties encountered in [2] will be avoided by reformulating the Eqs. (1.1) and (1.3) so as to eliminate the 'compressive' solutions. In this way it will be possible to prove the existence of a unique solution to (1.1) and (1.3) (under arbitrary loads) having $H+h>0$, and to construct a numerical procedure which converges to this solution regardless of the loads and physical characteristics of the suspension bridge.

2. The Solution. It was observed in the previous section that (1.1) is nonlinear due to the appearance in the equation of the induced live-load tension $h$. If $h$ were known, (1.1) would be a linear equation with constant coefficients and could be solved by standard techniques. Thus the objective will be to eliminate $w$ between Eqs. (1.1) and (1.3)-leaving a single equation for $h$. For this purpose (1.1) may be integrated twice to yield

$$
E I \frac{d^{2} w}{d x^{2}}-(H+h) w=A+B x+\int_{0}^{x} \int_{0}^{\omega}\left\{P(\tau)-\frac{h}{H} Q(\tau)\right\} d \tau d \omega
$$

where $A$ and $B$ are constants to be determined from the boundary conditions (1.2). Equation (2.1) may be simplified by an integration by parts. The result is

$$
\begin{aligned}
E I \frac{d^{2} w}{d x^{2}}-(H+h) w= & A+B x+x \int_{0}^{x}\left\{P(\tau)-\frac{h}{H} Q(\tau)\right\} d \tau \\
& -\int_{0}^{x} \tau\left\{P(\tau)-\frac{h}{H} Q(\tau)\right\} d \tau .
\end{aligned}
$$

It is easily shown that the boundary conditions (1.2) and Eq. (2.2) imply that $A=0$ and

$$
B=-\int_{0}^{l}\left\{P(\tau)-\frac{h}{H} Q(\tau)\right\} d \tau+\frac{1}{l} \int_{0}^{l} \tau\left\{P(\tau)-\frac{h}{H} Q(\tau)\right\} d \tau,
$$

so that (2.2) becomes

$$
\begin{aligned}
E I \frac{d^{2} w}{d x^{2}}-(H+h) w= & -\int_{0}^{x} \frac{(l-x) \tau}{l}\left\{P(\tau)-\frac{h}{H} Q(\tau)\right\} d \tau \\
& -\int_{x}^{l} \frac{x(l-\tau)}{l}\left\{P(\tau)-\frac{h}{H} Q(\tau)\right\} d \tau
\end{aligned}
$$

or, in simpler notation,

$$
\frac{d^{2} w}{d x^{2}}-\frac{(H+h) w}{E I}=-\frac{1}{E I} \int_{0}^{l} K(x, \tau)\left\{P(\tau)-\frac{h}{H} Q(\tau)\right\} d \tau
$$

where

$$
\begin{aligned}
K(x, \tau) & =(l-x) \tau / l, & & 0 \leqq \tau \leqq x \\
& =x(l-\tau) / l, & & x \leqq \tau \leqq l .
\end{aligned}
$$


The Green's function for (2.4) with a given $h$ will have one of two forms depending on whether $H+h>0$ or $H+h<0$. Thus in the case $H+h>0$ the Green's function becomes (cf. [3, pp. 351ff])

$$
\begin{aligned}
G_{+}(x, \xi ; h) & =\frac{\sinh \lambda \xi \sinh \lambda(l-x)}{\lambda \sinh \lambda l}, & & 0 \leqq \xi \leqq x, \\
& =\frac{\sinh \lambda x \sinh \lambda(l-\xi)}{\lambda \sinh \lambda l}, & & x \leqq \xi \leqq l .
\end{aligned}
$$

and in the case $H+h<0$

$$
\begin{aligned}
G_{-}(x, \xi ; h) & =\frac{\sin \lambda \xi \sin \lambda(l-x)}{\lambda \sin \lambda l}, & & 0 \leqq \xi \leqq x, \\
& =\frac{\sin \lambda x \sin \lambda(l-\xi)}{\lambda \sin \lambda l}, & & x \leqq \xi \leqq l .
\end{aligned}
$$

In both (2.6) and (2.7)

$$
\lambda=(|H+h| / E I)^{1 / 2} .
$$

The effect of the distinct Green's functions (2.6) and (2.7) is to permit a division of the problem into two parts - one of which corresponds to a tension in the cable and the other to a compression. Since, as was indicated previously, the solution corresponding to a tension in the cable (i.e. $H+h>0$ ) is the reasonable choice, the vertical deflection $w$ may be written

$$
w=\frac{1}{E I} \int_{0}^{l}\left\{G_{+}(x, \xi ; h) \int_{0}^{l} K(\xi, \tau)\left(P(\tau)-\frac{h}{H} Q(\tau)\right) d \tau\right\} d \xi .
$$

This expressi in for $w$ may be used with (1.3) to find an equation for $h$, i.e.

$$
\begin{aligned}
h & =\frac{E_{c} A_{c}}{E I H l_{c}} \int_{0}^{l}\left[Q(x) \int_{0}^{l}\left\{G_{+}(x, \xi ; h) \int_{0}^{l} K(\xi, \tau)\left(P(\tau)-\frac{h}{H} Q(\tau)\right) d \tau\right\} d \xi\right] d x \\
& =F(h) .
\end{aligned}
$$

It is clear that any solution of Eqs. (1.1) and (1.3) having $H+h>0$ and satisfying the boundary conditions (1.2) is a solution of (2.9) and (2.10). Conversely any solution of (2.9) and (2.10) is a solution of (1.1) and (1.3) and satisfies the boundary conditions (1.2). Moreover, any solution of (2.10) has $h>0$ (so that $H+h>0)$. To show this it is only necessary to note that $G_{+}(x, \xi ; h) \geqq 0$ and $K(\xi, \tau) \geqq 0$ (recall that $P$ and $Q$ are positive downward), so that $h<0$ implies that $F(h)>0$ (cf. (2.10)). Hence (2.10) cannot have a solution with $h<0$.

It is equally easy to show that there is a solution of $(2.10)$ for $h>0$. Consider the two curves defined by

$$
\begin{aligned}
& y=h, \\
& y=F(h),
\end{aligned}
$$

and note that an intersection of these two continuous curves corresponds to a solution of (2.10). However, at $h=0, F(h)>0$ while it is clear that $F(h)<0$ if $h$ is sufficiently large (cf. (2.10)). Thus the two curves do intersect and a solu- 
tion of (2.10) certainly exists. This result may be restated as

Theorem. The Eqs. (1.1) and (1.3) with boundary condition (1.2) have a solution such that $H+h>0$.

In the preceding discussion it has been shown that there exists a solution of (1.1) and (1.3) having $h>0$. It will now be shown that this solution is unique if the horizontal dead-load tension $H$ is sufficiently large. It is convenient to begin by discussing the Green's function (2.1).

Lemma. $G_{+}(x, \xi ; h)$ and $\left|\partial G_{+}(x, \xi ; h) / \partial h\right|$ are nonincreasing functions of $h$ and $H$ for all $x$ and $\xi$ in the interval $(0, l)$.

Proof. Let $f(x)$ be any nonnegative function. The solution of the differential equation

$$
W^{\prime \prime}-(H+h) W / E I=-f(x)
$$

with boundary conditions (1.2a) may be written in the form

$$
W=\int_{0}^{l} G_{+}(x, \xi ; h) f(\xi) d \xi .
$$

From the representation (2.13) it follows that $W(x ; h) \geqq 0$ for $0 \leqq x \leqq l$ and that $W_{h}(x ; h)$, the derivative of $W$ with respect to $h$, exists. Since $W$ is a solution of (2.12) with boundary conditions (1.2a), $W_{h}$ must be a solution of

$$
W_{h}^{\prime \prime}-(H+h) W_{h} / E I=W / E I
$$

with boundary conditions (1.2a). Thus $W_{h}$ may be written in the form

$$
W_{h}=-\frac{1}{E I} \int_{0}^{l} G_{+}(x, \xi ; h) W(\xi ; h) d \xi .
$$

Since $W(x ; h)$ is nonnegative $(2.15)$ implies that $W_{h}(x ; h) \leqq 0$ if $0 \leqq x \leqq l$, or from $(2.13)$

$$
W_{h}=\int_{0}^{l} \frac{\partial}{\partial h} G_{+}(x, \xi ; h) f(\xi) d \xi \leqq 0
$$

if $0 \leqq x \leqq l$. Since the inequality (2.16) holds for all nonnegative functions $f(x)$ it follows that

$$
\partial G_{+}(x, \xi ; h) / \partial h \leqq 0
$$

if $x$ and $\xi$ are in the interval $(0, l)$. In order to discuss the behavior of $\left|\partial G_{+}(x, \xi ; h) / \partial h\right|$ it suffices to compare (2.15) and (2.16). Thus

$$
\int_{0}^{l}\left\{\frac{\partial}{\partial h} G_{+}(x, \xi ; h) f(\xi)+\frac{1}{E I} G_{+}(x, \xi ; h) W(\xi ; h)\right\} d \xi=0
$$

for all nonnegative functions $f(x)$. Using (2.13) and interchanging the order of integration it is found that

$$
\int_{0}^{l}\left\{\frac{\partial}{\partial h} G_{+}(x, \xi ; h)+\left[\frac{1}{E I} \int_{0}^{l} G_{+}(x, \tau ; h) G_{+}(\xi, \tau ; h) d \tau\right]\right\} f(\xi) d \xi=0 .
$$

In view of (2.18) (recall that $f(x)$ is an arbitrary nonnegative function) it is clear that 


$$
\frac{\partial}{\partial h} G_{+}(x, \xi ; h)=-\frac{1}{E I} \int_{0}^{l} G_{+}(x, \tau ; h) G_{+}(\xi, \tau ; h) d \tau .
$$

Since $G_{+}(x, \xi ; h)$ is a nonincreasing function of $h$ it follows that $\left|\partial G_{+}(x, \xi ; h) / \partial h\right|$ is a nonincreasing function of $h$ and, hence, is bounded. In view of the way $H$ occurs in Eq. (2.14) it is clear that the preceding remarks also hold when $G_{+}(x, \xi ; h)$ is treated as a function of $H$. This completes the proof of the lemma.

The function $P(x)-h Q(x) / H$ (cf. (2.10)) is a monotone decreasing function of $h$. Therefore the right side of (2.10) is monotone decreasing as long as $P(x)-h Q(x) / H \geqq 0$. For example, if $P$ and $Q$ are constant $F(h)=0$ (cf. (2.10)) at $h=H P / Q$. If $h<H P / Q$ the lemma implies that $F(h)$ is monotone decreasing, and if $h>H P / Q$ the function $F(h)$ is negative. Since $h$, i.e. the left side of (2.10), is positive and monotone increasing it is clear that

Corollary. If $P$ and $Q$ are constant the Eqs. (1.1) and (1.3) with boundary conditions (1.2) have exactly one solution satisfying the condition $H+h>0$.

If the functions $P(x)$ and $Q(x)$ are not constant there will in general be a range of $h$ for which $P(x)-h Q(x) / H$ oscillates about zero. For $h$ in this range it is not clear that $F(h)$ is monotone. Thus it is necessary to proceed in a different manner.

In order to simplify the notation introduce

$$
L(H, h, \gamma)=\frac{E_{c} A_{c}}{E I H l_{c}} \int_{0}^{l}\left[Q(x) \int_{0}^{l}\left\{G_{+}(x, \xi ; h) \int_{0}^{l} K(\xi, \tau) \gamma(\tau) d t\right\} d \xi\right] d x .
$$

In this notation $(2.10)$ becomes

$$
h=L(H, h, P)-h L(H, h, Q) / H
$$

or

$$
h=\frac{L(H, h, P)}{1+L(H, h, Q) / H}=M(H, h, P, Q) .
$$

The object now will be to show that the slope of the right side of $(2.20)$ is less than one. From this fact the uniqueness of the solution of (2.20) (and hence (2.10)) would follow. Upon differentiation of (2.20) it is found that

$$
\begin{aligned}
\frac{\partial M(H, h, P, Q)}{\partial h}= & \frac{\frac{\partial}{\partial h} L(H, h, P)+\frac{L(H, h, P)}{H} \frac{\partial}{\partial h} L(H, h, P)}{\left(1+\frac{1}{H} L(H, h, Q)\right)^{2}} \\
& -\frac{\frac{L(H, h, P)}{H} \frac{\partial}{\partial h} L(H, h, Q)}{\left(1+\frac{1}{H} L(H, h, Q)\right)^{2}} .
\end{aligned}
$$

The first term on the right of (2.21) is negative. Therefore it suffices to show that the second term is less than one. Because of the monotonicity properties of $G_{+}(x, \xi ; h)$ and $\left|\partial G_{+}(x, \xi ; h) / \partial h\right|$ it is clear that

$$
\left|\frac{L(H, h, P)}{H} \frac{\partial}{\partial h} L(H, h, Q)\right| \leqq\left|\frac{L(H, 0, P)}{H} \frac{\partial}{\partial h} L(H, 0, Q)\right| .
$$


In addition $L(H, 0, P)$ and $|\partial L(H, 0, Q) / \partial h|$ are nonincreasing functions of $H$. Thus it is possible to choose $H$ so large that

$$
(1 / H)|L(H, 0, P)(\partial L(H, 0, Q)) / \partial h|<1 .
$$

Since the denominator of (2.21) is clearly greater than one it follows that for $H$ sufficiently large $\partial M(H, h, P, Q) / \partial h<1$ and hence the solution of $(2.20)$ is unique. Therefore

Theorem. For all functions $P(x)$ and $Q(x) \quad(\geqq 0)$ there exists a number $H^{*}$ such that $H>H^{*}$ implies that the solution of (2.10) is unique.

Since the preceding remarks guarantee that the solution of $(2.10)$ will have $H+h>0$ it only remains to construct a numerical procedure for the solution of (2.10) - or equivalently a procedure for the solution of the simultaneous equations (2.11). For this purpose the method of false position, Regula Falsi, was employed. Thus (2.11b) was evaluated for two values of $h$, e.g. $h_{0}$ and $h_{1}$, so that $y_{0}=F\left(h_{0}\right)$ and $y_{1}=F\left(h_{1}\right)$. A new value of $h$, say $h_{2}$, was chosen as the point of intersection of the curve (2.11a) and the chord connecting $\left(h_{0}, y_{0}\right)$ and $\left(h_{1}, y_{1}\right)$. The equation of the chord is given by

$$
y=y_{1}+\frac{y_{1}-y_{0}}{h_{1}-h_{0}}\left(h-h_{0}\right)
$$

so that $h_{2}$, the value of $h$ for which (2.22) and (2.11a) intersect becomes

$$
h_{2}=\frac{y_{0} h_{1}-h_{0} y_{1}}{\left(h_{1}-h_{0}\right)-\left(y_{1}-y_{0}\right)} .
$$

This process was then repeated, i.e. $h_{3}$ was computed as the point of intersection of the curve (2.11a) and the chord connecting $\left(h_{1}, y_{1}\right)$ and $\left(h_{2}, y_{2}\right)$ where $y_{2}=F\left(h_{2}\right)$. In general this scheme may be written

$$
h_{n+1}=\frac{y_{n-1} h_{n}-h_{n-1} y_{n}}{\left(h_{n}-h_{n-1}\right)-\left(y_{n}-y_{n-1}\right)}
$$

and

$$
y_{n+1}=F\left(h_{n+1}\right) \text {. }
$$

The iteration procedure (2.23) and (2.24) was actually applied to a suspension bridge having approximately the same physical characteristics as the center span of the Throgs Neck Bridge (the exact data for the Throgs Neck Bridge is given in [2]). In order to start the iteration procedure $h_{0}$ was chosen as $h_{0}=0$ and different choices were made for $h_{1}$ varying from $h_{1}=10 \mathrm{lbs} / \mathrm{ft}^{2}$ to $h_{1}=1 \times 10^{6} \mathrm{lbs} / \mathrm{ft}^{2}$ (the actual solution for the data used was $\left.h=2.4891 \times 10^{5} \mathrm{lbs} / \mathrm{ft}^{2}\right)$. In all cases it was found that the iteration scheme converged in no more than five iterations to within an error of $4 \times 10^{-5}$ percent. Thus the scheme converged rapidly even though the choice for $h_{1}$ was not close to the actual solution.

University of Wisconsin

Madison, Wisconsin

1. A. Pugstey, The Theory of Suspension Bridges, Edward Arnold, London, 19:57.

2. J. Heller, E. IsAaCson \& J. J. Stoker, The Numerical Solution of the Multispan Suspension Bridge Deflection Equations. (To appear.)

3. R. Courant \& D. Hilbert, Methods of Mathematical Physics, Vol. 1, Interscience, New York, 1953. MR 16, 426. 